\title{
Transcatheter or surgical aortic valve replacement for patients with severe, symptomatic, aortic stenosis at low to intermediate surgical risk: a clinical practice guideline
}

(c) $\frac{(1)(8)}{10}$ OPEN ACCESS

In patients with symptomatic severe aortic stenosis but at lower risk of perioperative death, how do minimally invasive techniques compare with open surgery? Prompted by a recent trial, an expert panel produced these recommendations based on three linked rapid systematic reviews

Per O Vandvik associate professor ${ }^{12}$, Catherine M Otto professor ${ }^{3}$, Reed A Siemieniuk methodologist ${ }^{4}$, Rodrigo Bagur assistant clinical professor ${ }^{6}$, Gordon H Guyatt distinguished professor $^{47}$, Lyubov Lytvyn methodologist ${ }^{8}$, Richard Whitlock associate professor ${ }^{9}{ }^{10}$, Trond Vartdal consultant physician ${ }^{11}$, David Brieger professor ${ }^{12}$, Bert Aertgeerts professor ${ }^{13}$, Susanna Price professor $^{14}$, Farid Foroutan graduate student ${ }^{415}$, Michael Shapiro community representative and senior health informaticist for RTI International ${ }^{16}$, Ray Mertz community representative ${ }^{17}$, Frederick A. Spencer professor ${ }^{4}$

\footnotetext{
${ }^{1}$ Institute of Health and Society, Faculty of Medicine, University of Oslo, 0318 Oslo, Norway; ${ }^{2}$ Department of Medicine, Innlandet Hospital Trust-division, Gjøvik, Norway; ${ }^{3}$ Division of Cardiology, Department of Medicine, University of Washington School of Medicine, Seattle, Washington, USA 98195; ${ }^{4}$ Department of Clinical Epidemiology and Biostatistics, McMaster University, Hamilton, Ontario, Canada L8S 4L8; ${ }^{5}$ Department of Medicine, University of Toronto, Toronto, Ontario, Canada; ${ }^{6}$ Division of Cardiology, London Health Sciences Centre and Department of Epidemiology \& Biostatistics, Western University, London, Ontario, Canada N6A 3K7; ${ }^{7}$ Department of Medicine, McMaster University, Hamilton, Ontario, Canada L8S 4L8; ${ }^{8}$ Systematic Overviews through advancing Research Technology, Child Health Evaluative Sciences, The Hospital for Sick Children Research Institute, Toronto, Ontario Canada M5G 0A4; ${ }^{9}$ Department of Surgery, McMaster University, Hamilton, Ontario, Canada L8S 4L8; ${ }^{10}$ Population Health Research Institute, Hamilton, Ontario, Canada L8L 2X2; ${ }^{11}$ Department of Cardiology, Oslo University Hospital - Rikshospitalet, 0424 Oslo, Norway; ${ }^{12}$ Concord Repatriation General Hospital, Concord, New South Wales 2139, Australia; ${ }^{13}$ Department of Public Health and Primary Care, KU Leuven, 3000 Leuven, Belgium; ${ }^{14}$ Royal Brompton Hospital, London SW3 6NP, UK; ${ }^{15}$ Heart Failure/Transplant Program, Toronto General Hospital, University Health Network, Toronto, Ontario, Canada M5G 2C4; ${ }^{16}$ Chicago, Illinois, USA; ${ }^{17}$ London, Ontario, Canada
} 


\section{Choice of intervention for those with severe aortic stenosis}

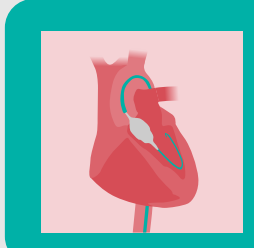

Transfemoral TAVI

Inserting a new valve into the

aortic valve's place without

open heart surgery. Delivery

is through the femoral artery.

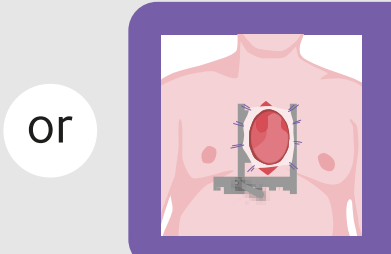

\section{SAVR}

Open-heart surgery, to remove

the narrowed aortic valve.

Replacement with tissue valve.

\section{Recommendations}

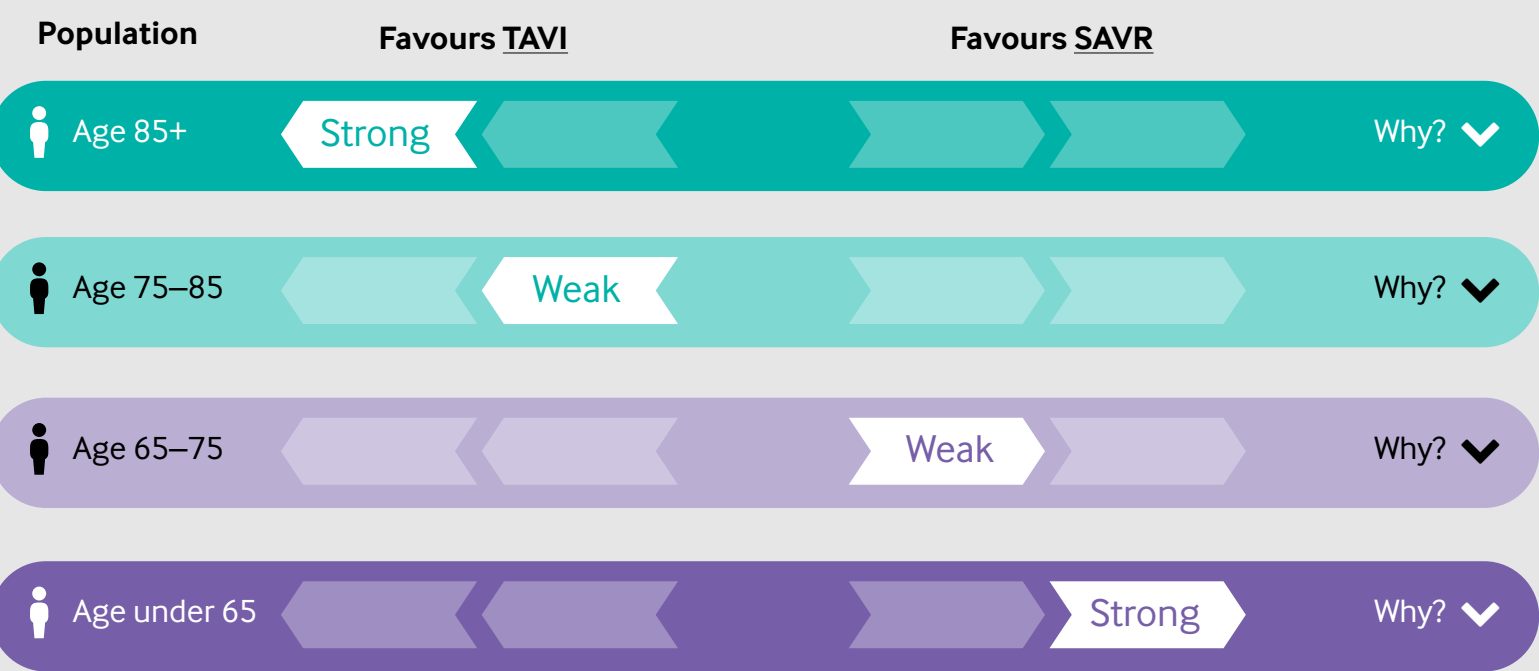

\section{Key uncertainties}

The major uncertainty is the durability of TAVI valves which drives recommendations in favour of SAVR in younger patients.
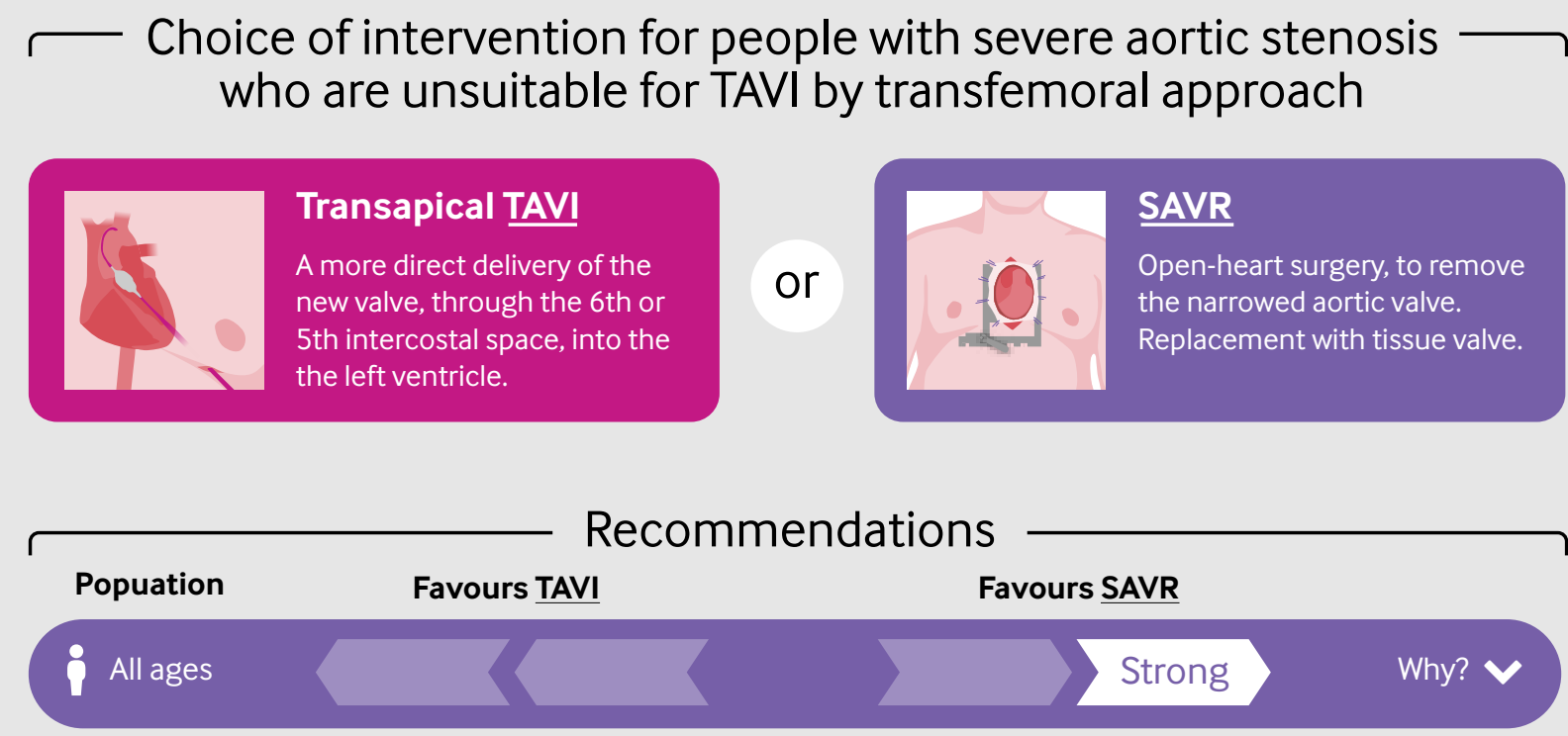

For a fully interactive version of this graphical summary of recommendations, please visit: https://doi.org/10.1136/bmj.i5085 
A randomised controlled trial of transcatheter aortic valve insertion (TAVI) versus surgical aortic valve replacement (SAVR) in patients with severe aortic stenosis was published in April 2016. The Partner 2 trial included 2032 people at intermediate surgical risk and favoured TAVI over open SAVR at two years for some outcomes. ${ }^{1}$ It had the potential to change practice.

Before the availability of TAVI, the only effective treatment for symptomatic severe aortic stenosis was SAVR with mechanical or bioprosthetic valves (fig $1 \Downarrow$ ). In practice, patients offered mechanical valves tend to be younger and must accept lifelong anticoagulation. The minimally invasive option, TAVI, was developed for patients who are unfit for surgery, in whom its use is recommended by major US and European guidelines. ${ }^{23}$

Severe aortic stenosis affects approximately 3 in 100 people over the age of 75 years. ${ }^{4}$ Patients typically experience symptoms of heart failure and reduced quality of life. Without aortic valve replacement, life expectancy is typically $50 \%$ at two years, with escalation of heart failure and reduced quality of life. ${ }^{5}$ These recommendations are for patients with symptoms and severe aortic stenosis: patients without symptoms or with milder disease are not considered here. Box 1 shows the linked articles in this BMJ Rapid Recommendations cluster.

\section{How the recommendations were created}

The international expert panel included front line and intervention cardiologists, a heart surgeon, internists, a general practitioner, methodologists, and people with lived experience of the condition (see list of panel members). To avoid bias, we excluded people with a professional, academic, or financial conflict of interest that we judged as excessive (see online methods supplement, competing interests statement, and appendix of interests of the panel members).

The panel followed standards for trustworthy guidelines and used the GRADE methodology to critically appraise the evidence and to create recommendations. ${ }^{6-8}$ When moving from evidence to recommendations, the panel integrated information on benefits and harms of treatment alternatives, quality of evidence, and values and preferences of patients as well as acceptability, feasibility, and resources (see box 1). Estimates of absolute effects are derived from relative effect estimates from the systematic review coupled with the best population estimates of baseline risks derived from a linked systematic review of observational studies. ${ }^{9}$ We label recommendations for or against each alternative as either weak or strong (see infographic).

\section{The evidence}

A linked systematic review and meta-analysis combines the data from the Partner 2 trial with three other trials (see fig $2 \Downarrow$ ). It pools data on 3128 patients with symptomatic severe aortic stenosis at low or moderate risk of perioperative death, typically followed for two years. ${ }^{1-14}$

Compared with SAVR, transfemoral TAVI reduced mortality and stroke, life threatening bleeds, atrial fibrillation, and acute kidney injury at two years, but increased heart failure, major vascular complications, pacemaker insertion, and need for aortic valve reintervention within 2 years (see infographic)..$^{10}$ In contrast, transapical TAVI may increase mortality and stroke compared with the surgical approach. The results for mortality, stroke, and acute kidney injury were based on a subgroup analysis for transfemoral versus transapical approach, deemed credible by the review authors according to specific criteria. ${ }^{15}$

Estimates of baseline risk for most outcomes came from a linked systematic review on prognosis for patients undergoing SAVR. ${ }^{9}$

The panel were reasonably confident that the absolute effect sizes for the short term benefit and harm of TAVI and SAVR were true and accurate estimates (that is, overall moderate certainty according to GRADE). But the panel were very uncertain about the long term durability of the valves used in TAVI, particularly with respect to their recommendations in younger patients with a longer predicted life expectancy. The low certainty in the estimated long term re-intervention rate after TAVI of approximately 27 in 100 people reflects an absence of published follow-up studies beyond five years.

The linked systematic review on values and preferences yielded extremely limited evidence to inform the recommendations, particularly their strength. ${ }^{16}$ There are no studies on patients deciding between TAVI and SAVR, although one study evaluated patient preferences and values when deciding whether to undergo SAVR (versus no surgery). ${ }^{16}$ One study identified multiple biomedical, functional, social, and environmental factors influencing patients' decisions to undergo assessment for TAVI.$^{18}$ Overall, there is evidence of variability in individual values and preferences, highlighting the need for shared decision making, particularly for patients aged between 65 and 85 years.

\section{Practical considerations}

Perioperative risk is typically assessed by expert cardiovascular teams. Validated risk scores such as the STS-PROM are available online (www.riskcalc.sts).

Type of device and TAVI approach may vary: balloon expandable and self expanding devices can be used via

This BMJ Rapid Recommendations article is one of a series that provides clinicians with trustworthy recommendations for potentially practice changing evidence. BMJ Rapid Recommendations represent a collaborative effort between the MAGIC group (www.magicproject.org) and The BMJ. A summary is offered here and the full version including decision aids is on the Magic app (www.magicapp.org), for all devices in multilayered formats. Those reading and using these recommendations should consider individual patient circumstances such as frailty, overall life expectancy, and relevant comorbidity, and their values and preferences. We encourage adaptation of recommendations to allow contextualisation of recommendations and to reduce duplication of work. Those considering use or adaptation of content may go to MAGICapp to link or extract its content or contact The BMJ for permission to reuse content in this article. Series adviser Rafael Perera-Salazar 


\section{What you need to know}

- New trial evidence confirms that transcatheter aortic valve insertion (TAVI), initially developed for patients with severe aortic stenosis who were unfit for surgical aortic valve replacement (SAVR), can be offered also to patients at low to intermediate surgical risk

- Long term durability of TAVI valves remains highly uncertain

- Age stratified recommendations reflect that TAVI is probably preferable to those over 75 years old, whereas SAVR is likely preferable to those under 75 years

\section{Box 1: Linked articles in this $B M J$ Rapid Recommendations cluster}

- Siemieniuk RA, Agoritsas T, Manja V, et al. Transcatheter versus surgical aortic valve replacement in patients with severe aortic stenosis at low and intermediate risk: systematic review and meta-analysis. BMJ 2016;354:i5130. doi:10.1136/bmj.i5130

- Foroutan F, Guyatt GH, O'Brien K, et al. Prognosis after surgical replacement with a bioprosthetic aortic valve in patients with severe symptomatic aortic stenosis: systematic review of observational studies. BMJ 2016;354:i5065. doi:10.1136/bmj.i5065

- Lytvyn L, Guyatt GH, Manja V, et al. Patient values and preferences on transcatheter or surgical aortic valve replacement therapy for aortic stenosis: a systematic review. BMJ Open 2016;6:e014327. doi:10.1136/bmjopen-2016-014327

- Editorial: Siemieniuk RA, Macdonald H, Agoritsas T, Guyatt GH, Vandvik PO. Introduction to BMJ Rapid Recommendations. BMJ 2016;354:i5191, doi:10.1136/bmj.i5191.

- Magic App (www.magicapp.org/public/guideline/aEeKpL)

- Expanded version of the results with multilayered recommendations, evidence summaries, and decision aids for use on all devices

transfemoral, subclavian, direct aortic, transcarotid, or transapical (balloon only) routes.

Fig $3 \Downarrow$ lists issues that may influence a person's choice of procedure. These are further detailed in MAGICapp within the consultation decision aids that provide all desirable and undesirable consequences of treatment options.

\section{Costs and resources}

There are no cost effectiveness data for patients at low to intermediate surgical risk considering TAVI versus SAVR. Both procedures are resource demanding and require an experienced cardiovascular team (table $1 \Downarrow$ ). In general, TAVI devices are more expensive than surgery, but this extra cost may be partially offset by a slightly shorter hospital stay and less need for post-discharge rehabilitation.

\section{Future research}

Future recommendations and guidelines would benefit from studies that answer the following questions

- Qualitative or survey study. What are the values and preferences of patients deciding between TAVI and SAVR, particularly with respect to uncertain durability of TAVI devices, the desire to avoid open heart surgery, and post-procedure pain and recovery time?

- What is the durability of the TAVI valves beyond five years?

\section{Competing interests: All authors have completed the BMJ Rapid} Recommendations interests form. The BMJ Rapid Recommendations team judged that no panel member declared financial, professional, or academic interests that precluded authorship. The declared interests for each panel member are attached. No panel members declared any financial conflicts of interest related to this clinical question, specifically no financial ties to the heart valve industry. D Brieger receives personal and institutional research funding from industry, none relevant for TAVI or SAVR (has received funding from Eli Lilly, Astra Zeneca, Sanofi Aventis, Boerhinger Ingelheim, Pfizer, Merck Sharp and Dohme, Bristol-Myers Squibb, and Bayer all related to antithrombotic or diabetes therapy). $\mathrm{R}$ Whitlock has received funding for consulting with Atricure and Armetheon (both focus on atrial fibrillation management). R Bagur is a primary investigator of a trial on anticoagulation in atrial fibrillation, sponsored by Bristol-Myers Squibb. R Whitlock performs SAVR. R
Bagur performs TAVI. CM Otto has co-chaired the American College of Cardiology guidelines on valvular heart disease, which includes TAVI, and is the UpToDate editor on the same topic. $R$ Whitlock is the section lead for the American College of Chest Physicians valve guidelines. S Price is a panel member of the European Cardiology Society guidelines on heart valves. RA Siemieniuk, GH Guyatt, PO Vandvik, CM Otto, R Bagur, F Foroutan, and L Lytvyn participated in writing the complementary systematic reviews that formed the evidence base for this guideline. No panel member has previously formally made statements favouring either option in low or intermediate perioperative risk patients. This article was edited by $\mathrm{H}$ MacDonald at The $B M J$ who had no relevant financial or intellectual interests.

1 Leon MB, Smith CR, Mack MJ, et al. PARTNER 2 Investigators. Transcatheter or Surgical Aortic-Valve Replacement in Intermediate-Risk Patients. N Engl J Med 2016;374:1609-20. doi:10.1056/NEJMoa1514616pmid:27040324.

2 Nishimura RA, Otto CM, Bonow RO, et al. ACC/AHA Task Force Members. 2014 AHA/ACC Guideline for the Management of Patients With Valvular Heart Disease: executive summary: a report of the American College of Cardiology/American Heart Association Task Force on Practice Guidelines. Circulation 2014;129:2440-92. doi:10.1161/CIR. 0000000000000029 pmid:24589852.

3 Vahanian A, Alfieri O, Andreotti F, et al. Joint Task Force on the Management of Valvular Heart Disease of the European Society of Cardiology (ESC) European Association for Cardio-Thoracic Surgery (EACTS). Guidelines on the management of valvular heart disease (version 2012). Eur Heart J 2012;33:2451-96. doi:10.1093/eurheartj/ehs109pmid: 22922415.

4 Osnabrugge RL, Mylotte D, Head SJ, et al. Aortic stenosis in the elderly: disease prevalence and number of candidates for transcatheter aortic valve replacement: a meta-analysis and modeling study. J Am Coll Cardiol 2013;62:1002-12. doi:10.1016/j. jacc.2013.05.015pmid:23727214.

5 Otto CM. Timing of aortic valve surgery. Heart 2000;84:211-8. doi:10.1136/heart.84.2. 211 pmid: 10908267.

6 Laine C, Taichman DB, Mulrow C. Trustworthy clinical guidelines. Ann Intern Med 2011;154:774-5. doi:10.7326/0003-4819-154-11-201106070-00011pmid:21646561.

7 Guyatt GH, Oxman AD, Vist GE, et al. GRADE Working Group. GRADE: an emerging consensus on rating quality of evidence and strength of recommendations. $B M J$ 2008:336:924-6. doi:10.1136/bmj.39489.470347.ADpmid:18436948.

8 Alonso-Coello P, Oxman AD, Moberg J, et al. GRADE Working Group. GRADE Evidence to Decision (EtD) frameworks: a systematic and transparent approach to making well informed healthcare choices. 2: Clinical practice guidelines. BMJ 2016;353:i2089. doi:10. 1136/bmj.i2089pmid:27365494.

9 Foroutan F, Guyatt GH, O'Brien K, et al. Prognosis after surgical replacement with a bioprosthetic aortic valve in patients with severe symptomatic aortic stenosis: systematic review of observational studies. BMJ 2016;354:i5065. doi:10.1136/bmj.i5065.

10 Siemieniuk RA, Agoritsas T, Manja V, et al. Transcatheter versus surgical aortic valve replacement in patients with severe aortic stenosis at low and intermediate risk: systematic review and meta-analysis. BMJ 2016;354:i5130. doi:10.1136/bmj.i5130.

11 Nielsen $\mathrm{HH}$, Klaaborg KE, Nissen $\mathrm{H}$, et al. A prospective, randomised trial of transapical transcatheter aortic valve implantation vs. surgical aortic valve replacement in operable elderly patients with aortic stenosis: the STACCATO trial. Eurolntervention 2012;8:383-9. doi:10.4244/EIJV8I3A58pmid:22581299.

12 Adams DH, Popma JJ, Reardon MJ, et al. U.S. CoreValve Clinical Investigators. Transcatheter aortic-valve replacement with a self-expanding prosthesis. N Engl J Med 2014;370:1790-8. doi:10.1056/NEJMoa1400590pmid:24678937.

13 Søndergaard L, Steinbrüchel DA, Ihlemann N, et al. Two-year outcomes in patients with severe aortic valve stenosis randomized to transcatheter versus surgical aortic valve replacement: the All-Comers Nordic Aortic Valve Intervention Randomized Clinical Trial. 


\section{How patients were involved in the creation of this article}

Two people with lived experience of severe aortic stenosis were full panel members and contributed with their experience and perspectives throughout the Rapid Recommendations process. Initially the community panel members identified pain and recovery time (in addition to already identified outcomes) and practical issues as important for choosing between TAVI and SAVR. They also fully participated in teleconferences and email exchange to discuss the evidence, draft recommendations, and to provide feedback in the creation of this article.

Circ Cardiovasc Interv 2016;9:e003665. doi:10.1161/CIRCINTERVENTIONS.115. 003665 pmid:27296202.

14 Thyregod HG, Steinbrüchel DA, Ihlemann N, et al. Transcatheter versus surgical aortic valve replacement in patients with severe aortic valve stenosis: 1-year results from the All-Comers NOTION Randomized Clinical Trial. J Am Coll Cardiol 2015;65:2184-94. doi: 10.1016/j.jacc.2015.03.014pmid:25787196.

15 Sun X, Briel M, Walter SD, Guyatt GH. Is a subgroup effect believable? Updating criteria to evaluate the credibility of subgroup analyses. BMJ 2010;340:c117. doi:10.1136/bmj. c117pmid:20354011.

16 Lytvyn L, Guyatt GH, Manja V, et alPatient values and preferences on transcatheter or surgical aortic valve replacement therapy for aortic stenosis: a systematic review. BMJ Open 2016;6:e014327. doi:10.1136/bmjopen-2016-014327.

17 Lauck SB, Baumbusch J, Achtem L, et al. Factors influencing the decision of older adults to be assessed for transcatheter aortic valve implantation: An exploratory study. Eur $J$ Cardiovasc Nurs 2015;1474515115612927.pmid:26498908.
18 Hussain Al, Garratt AM, Brunborg C, Aakhus S, Gullestad L, Pettersen KI. Eliciting patient risk willingness in clinical consultations as a means of improving decision-making of aortic valve replacement. J Am Heart Assoc 2016;5:e002828. doi:10.1161/JAHA.115. 002828pmid:26994130.

Published by the BMJ Publishing Group Limited. For permission to use (where not already granted under a licence) please go to http://group.bmj.com/group/rights-licensing/ permissions

This is an Open Access article distributed in accordance with the Creative Commons Attribution Non Commercial (CC BY-NC 4.0) license, which permits others to distribute, remix, adapt, build upon this work non-commercially, and license their derivative works on different terms, provided the original work is properly cited and the use is non-commercial. See: http://creativecommons.org/licenses/by-nc/4.0/ 


\section{Table}

Table 1 | Facilities and teams required for transcatheter aortic valve insertion (TAVI) versus surgical aortic valve replacement (SAVR) ${ }^{\star}$

\begin{tabular}{|c|c|c|}
\hline \multirow[b]{2}{*}{ Resource } & \multicolumn{2}{|c|}{ Extent of requirement } \\
\hline & TAVI & SAVR \\
\hline Heart team & +++ & ++ \\
\hline Cardiac anaesthesia & +++ & +++ \\
\hline Hybrid suite & ++ & - \\
\hline Intensive care unit & ++ & +++ \\
\hline Coronary care unit & ++ & $+/-$ \\
\hline Length of hospital stay (days) $\dagger$ & $2-5$ & $5-10$ \\
\hline Rehabilitation & ++ & +++ \\
\hline
\end{tabular}

*Based on panel expert members experience from practice.

†Estimates reflect current practice according to content experts on the panel. Length of stay was considerably longer in the trials (see infographic). 


\section{Figures}

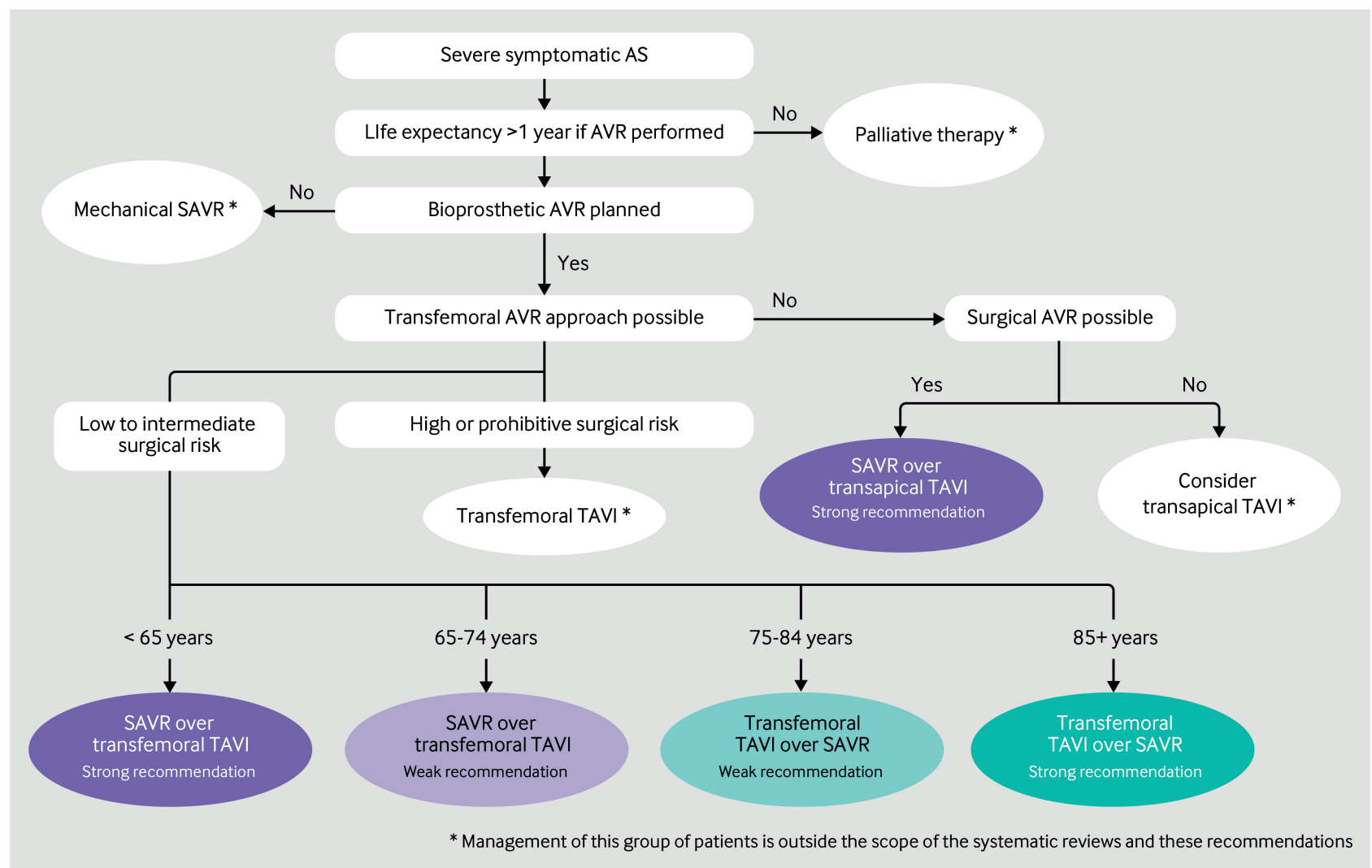

Fig 1 Flowchart for management of severe aortic stenosis (AS). Coloured boxes represent the recommendations covered by this article. $A V R=$ aortic valve replacement, SAVR=surgical aortic valve replacement, $\mathrm{TAVI}=$ transcatheter aortic valve insertion 


\section{Patient and trial characteristics}
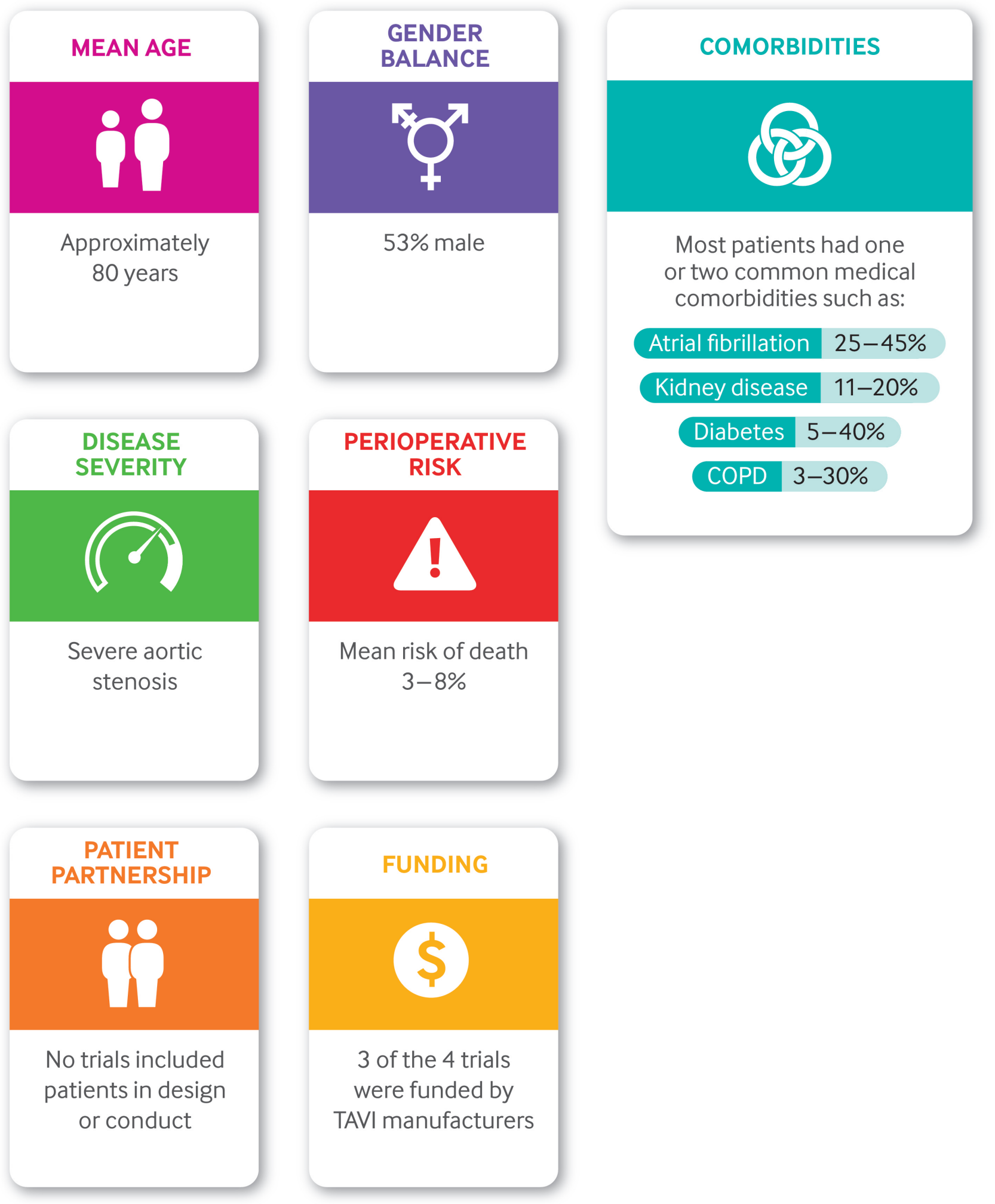

Fig 2 Patient and trial characteristics in the four trials of the linked meta-analysis ${ }^{10}$ 
PRACTICAL ISSUES

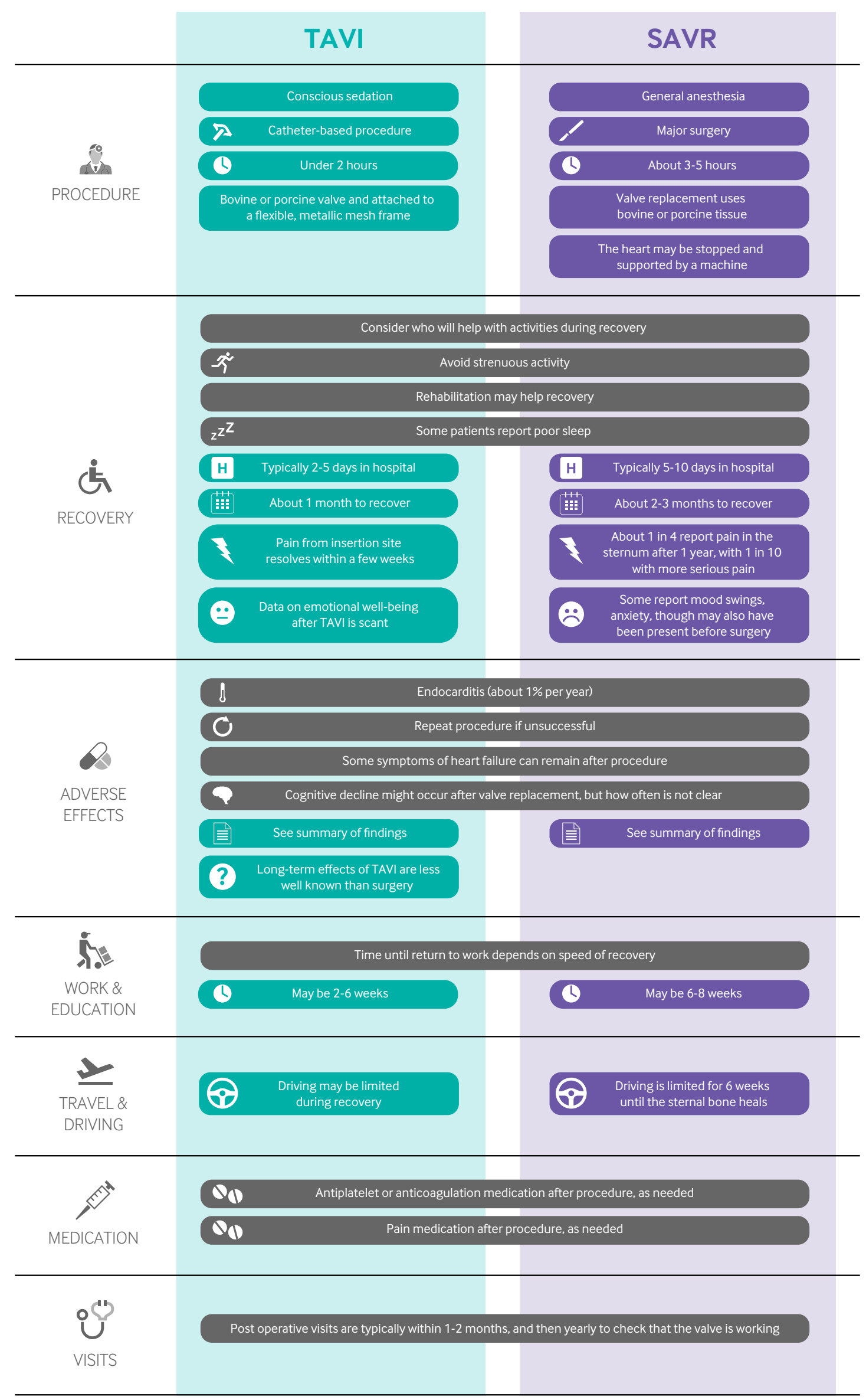

Fig 3 Practical considerations that may influence a patient's choice of procedure. For a complete list, see decision aids in the MAGICapp (www.magicapp.org/public/guideline/aEeKpL) 